\title{
El uso de las TIC y la formación permanente del adulto: una mejora de la calidad de vida
}

\author{
Paula Morales Almeida \\ Doctoranda de la Universidad de Las Palmas de Gran Canaria, España \\ pau_ma@hotmail.com
}

\begin{abstract}
UnESCO has insisted on various essential aspects to reinforce adult education in all countries in its various international conferences about it. In its Fifth Conference, unEsco confirms that information and communication technologies (ICT) are a powerful tool for people because it improves their control and gives them access to the most precious element in this XXI century: information. UnEsco deepens on the need for ongoing education training that must be done during a lifetime, not just during childhood and youth. Therefore, lifelong learning is one of the key points for a good functioning society. But it is also necessary that adults and elders acquire the digital competition so important nowadays, in order to make available to them a better quality of life. That way they may continue to be autonomous citizens. We must do not forget that ICT make our lives easier to live and are present in all day-to-day areas such as government, education, private enterprise and personal level. Consequently, if elders access to ICT in a responsible manner they will continue to be a part of society, so their life will have higher quality. We will see a group of women as case study. They noticed how their quality of life improved as they learned and applied the knowledge acquired in various computer classes. They finally found out that they can be an useful element of society and that they can keep learning new things, although they first thought that was impossible.
\end{abstract}

Keywords: ICT, adults, lifelong education, training, quality of life.

\section{Resumen}

La UNESCO en sus diferentes conferencias internacionales sobre la educación de adultos, ha incidido en diferentes aspectos esenciales para reforzarla en todos los países. En su quinta conferencia, la UnEsco confirma que las tecnologías de la información y la comunicación (en adelante TIC) son un instrumento poderoso para las personas porque incrementa su poder y les da acceso a la información, el bien más preciado en este siglo Xxi. Y profundiza en la necesidad de una educación permanente, una formación que se debe realizar durante toda la vida y ya no solo durante la infancia y juventud. De ahí que la educación permanente sea una de las grandes apuestas para este siglo. Pero también es necesario que las personas adultas y las mayores adquieran la competencia digital, tan importante en nuestros días, para que puedan tener una mejor calidad de vida, porque así podrán seguir siendo ciudadanos autónomos. No olvidemos que las TIC nos facilitan muchas de las tareas cotidianas de la vida y están presentes en todos los ámbitos, en las administraciones públicas, en las educativas, en la empresa privada y en el ámbito personal. Así, si los mayores acceden a las Tic de una manera responsable, seguirán siendo partícipes de esta sociedad, por lo que su vida tendrá una mayor calidad. Veremos el caso de un grupo de mujeres que vieron cómo su calidad de vida aumentaba a medida que conocían y aplicaban los conocimientos adquiridos en un curso de informática y comprobaron cómo aún son útiles en la sociedad y pueden seguir aprendiendo cosas nuevas, aunque ellas creyeran al principio que era imposible.

Palabras clave: TIC, adultos, educación permanente, formación, calidad de vida 


\section{Las TIC y los adultos}

Según la Unesco, en el capítulo 7 de la Quinta Conferencia Internacional sobre la Educación de las Personas Adultas, celebrado en Hamburgo en 1997, las tecnologías de la información y la comunicación (en adelante, TIC) son un instrumento poderoso que incrementa el poder de acceso de todo ciudadano y ciudadana a la información y a los nuevos métodos de educación, enriqueciendo su entorno de aprendizaje.

Las TIC nos abren un camino hacia la democratización del acceso a la educación, posibilitando el aprendizaje permanente. Un aprendizaje permanente que se hace cada vez más necesario dada la sociedad actual en la que vivimos, la sociedad de la información y la comunicación, y la esperanza de vida con la que cuentan los ciudadanos de los países desarrollados. La competencia digital, según Ortega Navas y Ortega Sánchez (2009: 122), «es la que conlleva un uso seguro y crítico de las tecnologías de la sociedad de la información (TSI) y por tanto, el dominio de las TIC».

Pero, para conseguir la competencia digital, tenemos que hablar primero de la alfabetización digital, que tendrá que tener en cuenta los contextos sociales y culturales de las personas, ya que no basta con enseñar a navegar por Internet, a enviar correos electrónicos o subir fotos en redes sociales. Se les debe enseñar a construir y elaborar conocimientos y a utilizar las Tic de un modo responsable. Se deberá tener en cuenta los conocimientos previos de las personas, el lugar donde viven, si poseen los recursos para poder acceder a dicha tecnología, etc. Ya que todos los adultos no tienen acceso ni a la información ni a las TIC, es lo que se conoce como brecha digital.

Martínez y Serrano (2003) afirman que la brecha digital es una separación que existe entre las personas (comunidades, estados, países...) que utilizan las tecnologías de la información y la comunicación (TIC) como una parte rutinaria de su vida diaria y aquellas que no tienen acceso a las mismas y que, aunque las tengan, no saben cómo utilizarlas. Cabero (2004) también señala que la brecha digital se está convirtiendo en un elemento de separación, de exclusión de personas, colectivos y países. El riesgo es que pasemos de una exclusión tecnológica a una exclusión social, personal y educativa. Es decir, que pasemos de una brecha digital a una brecha social, por lo que la tecnología no estaría propiciando la inclusión social de las personas, sino su exclusión del sistema. Y no debemos asumir dicho riesgo. Así, se debe acabar con la brecha digital que genera desigualdades y acercar las TIC a todos y no solo eso, sino que una vez que se obtiene el recurso hay que enseñar cómo utilizarlo; ahí contamos con la alfabetización digital. La brecha digital lleva a la exclusión digital, lo que debemos tener en cuenta es cómo podemos incluir a las personas en las TIC. La Unión Europea define la inclusión social de la siguiente manera (European Council 2004, citado por Ortoll, 2007: 31):

Proceso que garantiza que aquellos que están en riesgo de exclusión social obtienen las oportunidades y los recursos necesarios para participar de manera plena en la vida económica, social y cultural y que pueden gozar de un estilo de vida que se considera normal en la sociedad en que viven. Eso garantiza que tengan más participación en las decisiones que afectan a sus vidas y en el acceso a sus derechos fundamentales.

Así, debemos favorecer siempre la inclusión social y digital de las personas y, sobre todo, de los adultos y mayores que se encuentran más excluidos del sistema.

\section{El aprendizaje permantente}

La utilización de las Tic en el aprendizaje permanente no hace de la enseñanza una práctica despersonalizada ni mecanizada, sino todo lo contrario (Ortega Navas y Ortega Sánchez, (2009: 122).

...nos abre a un mundo encaminado hacia multitud de gente con formas de aprender distintas en la que la adquisición de la información y el conocimiento se hace de manera opcional y personal, un ordenador que se mantiene como nexo de unión entre la educación y la realidad, fomentando la creatividad y un humanismo creciente del que cada vez deberíamos ser más conscientes.

También la educación a distancia, a través de la red, nos abre nuevas posibilidades de aprendizaje a lo largo de toda la vida y facilita el acceso igualitario a la sociedad del conocimiento, ya que no hace 
falta la presencialidad en el aula, sino un ordenador desde el que podamos seguir la formación, lo que hace que aquellas personas que, por distintas razones, como puede ser una discapacidad, trabajo, obligaciones personales, no pueden acudir a un centro en horario ordinario puedan formarse en su casa y a su tiempo. Lo que en definitiva favorece la calidad de vida de las personas mayores, ya que, por un lado, gracias a las TIC, acceden a una formación permanente cada vez más necesaria y, gracias precisamente a las TIC, dicha formación la pueden realizar desde casa, por lo que aquellas personas que no pueden desplazarse al centro por diversos motivos seguirían optando a una educación de calidad como el resto de los ciudadanos que sí se pueden desplazar. Pero, para logar una verdadera inclusión de las personas mayores en la red, habrá que tener en cuenta una serie de cuestiones que hay que abordar sobre el acceso de los adultos a las TIC.

La primera de ellas son las cuestiones culturales, ya que, si queremos que las Tic lleguen a todos las personas y en especial a los adultos, se debe tener en cuenta su lengua materna, el acceso que puede llegar a tener cada uno, el lugar de procedencia, la importancia de que las organizaciones internacionales y las propias de cada país aúnen esfuerzos para favorecer el acceso a las Tic a toda la población, etc. Sin tener en cuenta las características de las personas es imposible lograr un verdadero acceso a las Tic.

Otra cuestión importante que hay que destacar es la educación a distancia, como ya hemos nombrado, ya que en los últimos años se ha incrementado el número de adultos que accede a la formación por medio de entornos virtuales. Estas personas acuden a clases virtualmente, pero muchas de ellas no tienen un verdadero acceso a la red, ya que no tienen la competencia digital adquirida; por lo tanto, es necesario primero alfabetizar digitalmente a aquellas personas que lo necesiten.

En este sentido, Área (2002, citado por Ortega Navas y Ortega Sánchez, 2009) orienta sobre la importancia de la alfabetización tecnológica de los adultos como factor de democratización, de formación de ciudadanos libres y críticos ante los nuevos medios de información.
Al mismo tiempo, las nuevas tecnologías refuerzan la disparidad social. Lo hemos comprobado anteriormente con la brecha digital, donde muchos adultos no consiguen acceder a las TIC y, por lo tanto, quedan excluidos de esta nueva sociedad de la información y el conocimiento.

El uso de Internet y de otras tecnologías de la información sigue estando dominado por las personas con un alto nivel de educación y de ingresos, porque el equipo a menudo no está al alcance de los demás y porque el analfabetismo tecnológico aún está muy difundido. Sin embargo, muchos países en desarrollo están probando las tecnologías de la información y la comunicación y creando nuevos sistemas de enseñanza abierta.

No menos importante es que las TIC aportarán una mejora de la calidad de vida de las personas mayores, ya que pueden reducir, y de hecho reducen, la sensación de aislamiento de las personas mayores, ya que en la red pueden establecer relaciones con muchísimas personas de otras partes del planeta, pueden aprender lo que siempre quisieron aprender pero que, por diversas razones, nunca pudieron estudiar. Su mente se ejercitará al estar conectados y navegando por la red. Su autoestima mejorará con las relaciones que establecerán y, lo más importante, volverán a ser partícipes de la sociedad, dando su valiosa experiencia y opinión. Por todo esto y mucho más, las Tic son hoy una poderosa herramienta para proporcionar a los mayores una mejora de su calidad de vida en todos los aspectos.

\section{La educación de adultos}

Se afirma que el siglo xxi será el siglo de la información y la educación se articula como uno de los pilares fundamentales por la importancia dada a la educación permanente; así, la educación de adultos tiene mucho que decir en este nuevo siglo, aunque aún se encuentra en proceso de transición (Área, 2000). La educación de adultos tendrá que hacer frente a una serie de necesidades nuevas que, como comenta Área (2000: 29), son:

- Los nuevos analfabetos: con los cambios acaecidos, los adultos han salido especialmente desfavorecidos, lo que ha provocado una ex- 
clusión de los adultos con respecto a las nuevas tecnologías.

- Mucha información pero poco conocimiento: tenemos mucha información a nuestro alcance, pero el adulto no está preparado para tratar esa información y convertirla en conocimiento.

- Fragmentación de la información: la publicación de la información a través de la red ha cambiado el sistema lineal que existía hasta entonces, por lo que los adultos pueden no ser capaces de captar toda la información que les llega.

- Desfase de los sistemas educativos: la educación siempre se ha caracterizado por la lentitud para introducir cambios en las escuelas, por ello las escuelas aún no están preparadas ni lo suficientemente dotadas con las tecnologías de la información y la comunicación.

- Nuevas exigencias de formación laborales: los cambios económicos y tecnológicos han modificado las estructuras laborales. La introducción de las TIC ha supuesto cambios en la manera de trabajar y comunicarse y ha supuesto la creación de nuevos puestos de trabajo.

En la educación de adultos encontramos una serie de principios básicos que es necesario conocer, (Área, 2000: 31-32). En primer lugar, hay que ver la educación de adultos como una educación para toda la vida, ya que la esperanza de vida cada vez es mayor y la sociedad de la información nos exige que estemos preparados ante los cambios que se producen cada día.

Por otro lado, la educación de adultos es una acción para el desarrollo democrático y cultural de nuestra sociedad, porque en esta nueva sociedad la participación es vital; la educación de adultos se puede ver como una estrategia de compensación de las desigualdades en el acceso al conocimiento, la educación de adultos es un derecho de todos los ciudadanos que implica la formación de aquellos ciudadanos que por diversos motivos, no pudieron formarse. Así, en esta nueva sociedad, la formación se les debe proporcionar sin ningún problema, ya que es un derecho básico. Por último, la educación de adultos debe dar respuesta a las nuevas necesidades y demandas sociolaborales, así, es nuestra labor formar a los nuevos ciudada- nos con respecto a estas necesidades que la sociedad requiere.

\section{Un caso práctico}

Para apoyar lo dicho anteriormente, se realizó un estudio con un grupo de mujeres que, durante el curso pasado, asistieron a un curso de informática y adquirieron la competencia digital. El curso se realizó en la asociación de mujeres Flora Tristán ubicado en el barrio de Jinámar en la isla de Gran Canaria. El perfil de estas señoras era el siguiente: mujeres, en su mayoría amas de casa, con un nivel económico y cultural bajo, ya que la mayor formación recibida era el graduado en educación secundaria y con unas edades comprendidas entre los 53 y los 76 años.

Por supuesto, no tenían adquirida ninguna competencia digital y ni siquiera estaban alfabetizadas digitalmente. Así, durante todo un curso, se les formó en un uso educativo del ordenador, abrieron sus propias cuentas de correo electrónico, conocieron diferentes redes sociales, aprendieron a utilizar programas como el Spyke (lo demandaban aquellas mujeres con hijos que vivían fuera de la isla), aprendieron cómo realizar presentaciones, videos, a escribir y modificar textos, a realizar sus propios escritos y currículos vítae, etc.

Cuando comenzaron el curso, se realizó un foro de discusión donde se discutieron diversas preguntas, entre ellas:

- ¿Por qué quiero aprender informática?

- ¿Qué creo que puede aportar a mi vida?

- ¿Qué me gustaría aprender?

- Lo que aprenda, ¿me ayudará a sentirme mejor conmigo misma?

Las respuestas a las diferentes preguntas fueron similares entre las mujeres. Todas deseaban aprender informática para sentirse más cerca de los familiares que sí sabían, para comunicarse con hijos y nietos, pero también para sentirse más útiles y poder aprender cómo pedir cita para el médico o para la renovación de documentación, buscar información básica sobre aquello que quieren saber sin necesidad de pedir un favor a nadie, etc. 
Tras esto, se procedió a realizar el curso durante todo un año. Y aprendieron muchísimas herramientas que eran capaces de aplicar en su vida diaria. Al término del curso, se volvió a realizar otro grupo de discusión donde se debatieron las siguientes preguntas:

- ¿He aprendido algo con el curso?

- Lo aprendido, ¿me ha favorecido a nivel personal, profesional, etc.?

Se realizaron diferentes sesiones, ya que las mujeres querían expresar cómo se habían sentido en esos meses que había durado el curso y contar cómo se sentían ahora. Todas se mostraban muy satisfechas por haber aprendido algo que consideraban que nunca hubieran podido aprender, por lo que su autoestima se vio completamente reforza- da. Lo que habían aprendido lo aplicaban a su vida diaria: ahora escribían cartas a amigas, se mandaban correos electrónicos y se comunicaban con sus hijos y nietos. Muchas de ellas actualizaban su currículo, pedían cita para el médico, para renovar el DNI, etc. Todas vieron cómo su calidad de vida aumentaba al adquirir la competencia digital y tras haber aprendido a dar un buen uso al ordenador y a Internet. Y querían saber más.

Así, es vital que nuestros adultos y mayores también adquieran la competencia digital y sepan hacer un buen uso de las TIC, ya que estas les pueden ayudar y de hecho les ayudan a mejorar su calidad de vida y volver a sentirse útiles en la sociedad, un sentimiento generalizado en muchas personas: volver a sentirse ciudadanos.

\section{Referencias}

Área, M. (2000). Redes virtuales para la educación de adultos. Una guía pedagógica. Un nuevo contexto para la educación de adultos: la sociedad de la información (pp. 19-38). Consultado el 1 de febrero del 2012 de: http://www. edulab.ull.es/www/02_publicaciones/01_redveda/capitulo1.pdf

Cabero, J. (2004). Formación del profesorado en TIC. II Congreso Nacional de Formación de Profesorado en Tecnologías de la Información y Comunicación. Jaén.

Mártinez, E. y Serrano, A. (2003). La brecha digital: mitos y realidades. México: UABC. Obtenido el día 4 de enero del 2004 de: http://www.labrechadigital.org/labrecha/index.php?option=com_content\&task=view\&id=118\&Itemid=41

Ortega, M. y Ortega, I. (2009). Nuevos contextos formativos para el desarrollo de la educación permanente. En LópezBarajas, E. El paradigma de la educación continua. Reto del siglo xxI, pp. 109-129. Madrid. Editorial Narcea.

Ortoll, E. (2007). La alfabetización digital en los procesos de inclusión social. Barcelona: Editorial uoc. 